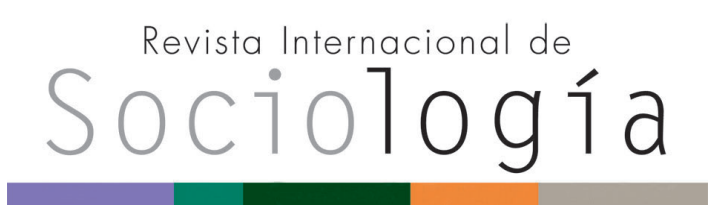

Revista Internacional de Sociología RIS vol. 73 (3), e020, septiembre-diciembre, 2015, ISSN-L:0034-9712

DOI: http://dx.doi.org/10.3989/ris.2015.73.3.e020

\section{RELIGIÓN E INSTITUCIONES PÚBLICAS EN ESPAÑA. Hospitales y prisiones en perspectiva comparada}

\author{
MAR GRIERA \\ Universitat Autònoma de Barcelona, España. \\ mariadelmar.griera@uab.com \\ Julia Martínez-Ariño \\ Max Planck Institute for the Study of Religious \\ and Ethnic Diversity, Alemania. \\ martineza@mmg.mpg.de
}

Como citar este artículo / Citation: Griera, M, MartínezAriño, J. A. Clot-Garrell \& García-Romeral, G. 2015. «Religión e instituciones públicas en España. Hospitales y prisiones en perspectiva comparada». Revista Internacional de Sociología, 73 (3) DOI: http://dx.doi.org/10.3989/ris.2015.73.3.e020

\section{Resumen}

A partir de una aproximación cualitativa, este artículo analiza las tensiones y negociaciones cotidianas que se producen en torno a la presencia y rol de la religión en las prisiones y hospitales contemporáneos en España. Más concretamente, el artículo examina la emergencia de nuevas formas de encaje entre la Iglesia católica, las minorías religiosas y las instituciones seculares; y sus implicaciones en cuanto a la naturaleza secular del Estado y las desigualdades entre grupos. La complejidad de las dinámicas religioso/secular, catolicismo/diversidad religiosa y religión/espiritualidad patentes en el marco de estas instituciones evidencia la imposibilidad de dibujar un modelo único para dar cuenta de cómo la religión y la diversidad religiosa son gestionadas en estos contextos.

\section{Palabras Clave}

Centros hospitalarios; Centros penitenciarios; Diversidad religiosa; Iglesia Católica; Secularización.

\section{RELIGION AND PUBLIC INSTITUTIONS IN SPAIN. A comparison of hospitals and prisons}

\author{
Anna Clot-Garrell \\ Universitat Autònoma de Barcelona, España. \\ Anna.clot@uab.cat \\ GLORIA Garcia-Romeral \\ Universitat Oberta de Catalunya, España. \\ ggarcia_romeral@uoc.edu
}

Copyright: @ 2015 CSIC. Este artículo de acceso abierto distribuido bajo los términos de la licencia Creative Commons Attribution-Non Commercial (by-cn) Spain 3.0.

Recibido: 21/11/2013. Aceptado: 27/09/2014

Publicación online: 10/11/2015

\section{Abstract}

Based on a qualitative approach, this article analyzes the current role of religion in Spanish prisons and hospitals. More specifically, the article explores the daily tensions and negotations between the Catholic Church, the religious minorities and the secular professionals working in these institutions. The complexity of the micro religious/secular dynamics, together with the mono/multiconfessional tension and the religious/spiritual divide going on within these institutions, demonstrate the impossibility of drawing a single model to account for the way in which religion is being dealt with in contemporary Spanish public institutions.

\section{KEYWORDS}

Catholic Church, Hospitals; Prisons; Religous Diversity; Secularization. 


\section{INTRODUCCIÓN}

Aún hoy se distingue lo que era la antigua entrada a la capilla del Centro Penitenciario de Hombres de Barcelona, la Modelo. Tiempo atrás eran frecuentes las celebraciones religiosas en este espacio, lugar donde oficiaba el capellán castrense de la prisión. La antigua capilla desapareció hace unos años y en su lugar se ubican dependencias administrativas y oficinas. Hoy en día los servicios religiosos se celebran en una pequeña sala que es también lugar de paso- en el interior del recinto penitenciario. La decoración es sencilla: unas cuantas sillas de plástico marrón y una mampara con candado que separa el altar católico del resto de la habitación -lo que permite que otras confesiones puedan también utilizar el espacio para sus actividades religiosas-. El caso del Centro Penitenciario de Hombres de Barcelona ilustra lo que es evidente a primera vista: la religión -y especialmente el catolicismo- ha perdido parte de la influencia que tenía antaño en el seno de las instituciones penitenciarias. La transición democrática -y la legislación subsiguiente en materia de libertad religiosa y de conciencia- marcó un hito importante en esta cuestión, desbancando formalmente a la Iglesia católica de su estatus oficial previo. Un proceso similar se ha producido en el ámbito sanitario, donde la religión católica ha ido perdiendo parte de su presencia e influencia; la retirada de cruces de las instalaciones de los hospitales o la limitación del acceso del capellán a todas las habitaciones son algunas de las muestras más visibles.

El impacto del proceso de secularización de la sociedad española (Pérez-Agote 2012) en sus múltiples niveles, pero de manera específica en términos de la separación IglesiaEstado, es claramente observable en el funcionamiento cotidiano de hospitales y prisiones. Sin embargo, no existen investigaciones que examinen cómo cristaliza el proceso de desconfesionalización en la práctica y cómo se reconfigura el rol de la religión en dichas instituciones en contextos concretos. Asimismo, los nuevos desafíos que genera el crecimiento de la diversidad religiosa también han pasado completamente desapercibidos en la bibliografía académica española, si bien este es un tema de creciente relevancia en el marco europeo. Así lo demuestran las investigaciones de James Beckford y Sophie Gilliat-Ray (1998) en Inglaterra, del mismo Beckford en colaboración con Danièle Joly y Farhad Khosrokhavar (2005) y de Corinne Rostaing, Claire de Galembert y Céline Béraud (2014) en Francia, de Inger Furseth en Noruega (2000; 2003; 2011), de Inés Michalowski (2010) en Alemania, de Valeria Fabretti (2014) en Italia y de Irene Becci (2011) en Italia, Alemania y Suiza. Desde enfoques teóricos diferentes, todos estos estudios analizan la manera como diversas instituciones públicas responden a la creciente diversidad religiosa, y cómo esta ha puesto en cuestión su funcionamiento, fuertemente ligado a las iglesias tradicionales.
Con la voluntad de contribuir al debate académico y suplir esta laguna, este artículo analiza el impacto que genera la transformación del escenario religioso en el seno de hospitales y prisiones en España. Según Wendy Cadge (2013), las instituciones públicas constituyen un espacio de análisis privilegiado para examinar las transformaciones actuales de la religión. La socióloga norteamericana afirma que investigar la religiosidad fuera del ámbito de las comunidades religiosas y de la vida individual del creyente es fundamental para comprender los procesos de reconfiguración de lo secular y lo religioso en la esfera pública contemporánea. De forma similar, Beckford y Gilliat-Ray (1998) argumentan la idoneidad de focalizar el análisis en prisiones y hospitales por el hecho de que, en ellos, los desafíos, conflictos y negociaciones de una sociedad multicultural y multirreligiosa pueden observarse como si fueran en miniatura. Las siguientes preguntas guían la reflexión: ¿Qué rol juega la religión en las prisiones y hospitales contemporáneos y cómo ha cambiado en los últimos años? ¿Cómo afrontan estas instituciones la creciente diversidad religiosa del país? ¿Cuáles son las mayores diferencias entre prisiones y hospitales en esta área y cómo las podemos explicar? A través de una aproximación empírica cualitativa, el artículo responde a estas cuestiones y se interroga sobre la emergencia de nuevas formas de encaje entre la Iglesia católica, las minorías religiosas y las instituciones seculares.

El artículo está estructurado en cuatro partes. La primera parte expone la aproximación teórica y metodológica. Los tres apartados siguientes exploran empíricamente la presencia de la religión en ambas instituciones, centrándose cada uno de ellos en una cuestión específica. El primero analiza el rol que juega la religión en las prisiones y hospitales contemporáneos, estudiando así la influencia y el alcance del proceso de separación Iglesia-Estado. El segundo apartado examina cómo estas instituciones responden a la creciente diversidad del país y cómo los profesionales perciben, valoran y actúan al respecto. El tercer apartado explora la emergencia de nuevas formas de religiosidad, poniendo el énfasis en las cuestiones que se ubican en la frontera entre lo religioso y lo espiritual. La comparación entre hospitales y prisiones es transversal a todo el artículo pero las conclusiones se centran en esta cuestión, buscando responder cuáles son las mayores diferencias entre ambas instituciones en esta área y cómo las podemos explicar.

\section{APROXIMACIÓN Y MÉTODOS: MÁS ALLÁ DE LOS MODELOS}

Teresa ${ }^{1}$ y el hermano Juan trabajan en el servicio religioso de un importante hospital gestionado por una orden religiosa

1 Todos los nombres de personas que aparecen en el artículo son nombres figurados. 
católica. Forman parte del equipo encargado de dar apoyo y acompañamiento espiritual a los pacientes, sus familias $y$, si se requiere, también al personal del centro. La semana después de Semana Santa de este año 2014 fuimos a entrevistarles. En el curso de nuestra conversación les preguntamos si habían hecho algo especial para celebrar la Semana Santa «más allá de no comer carne el Viernes Santo»-dando por supuesto que, siendo un hospital católico, la habrían eliminado del menú para aquel día-. Acto seguido se miraron el uno al otro y pocos segundos después el hermano Juan dijo: «Bien, en realidad, ellos [refiriéndose al personal de cocina] sirvieron albóndigas en Viernes Santo y cuando nos dimos cuenta ya era demasiado tarde». Y Teresa añadió: «Sí, incluso aquí [en un hospital católico] es realmente difícil conseguir que ellos se den cuenta de que la religión puede ser importante para algunas personas».

A primera vista esta anécdota podría hacernos pensar que la religión juega un papel irrelevante en las instituciones públicas y que el proceso de secularización ha desbancado completamente a la Iglesia católica. Sin embargo, unos meses antes vivimos una situación completamente opuesta en una entrevista con un trabajador social de una prisión recientemente inaugurada. En este caso, el trabajador social -que, además, en el transcurso de la entrevista nos dijo que se consideraba fuertemente ateo- nos explicó que él y el resto del equipo directivo habían tomado la decisión, como política general de la institución, de no servir carne a nadie (católicos y no católicos) el Viernes Santo.

¿Qué nos dice esto acerca del papel de la religión en estas dos instituciones? ¿Significa que las prisiones tienen una política más favorable a la presencia de la religión que los hospitales? A primera vista parecería que así es. Sin embargo, si en lugar de fijarnos en este episodio concreto dirigiésemos la atención a las salas de oración, podríamos contar una historia diferente. Así, mientras que en esta misma prisión recientemente inaugurada no hay un lugar específico para celebrar los servicios religiosos (solo un espacio multiusos que normalmente está ocupado con actividades recreativas o educativas), el hospital ya mencionado ha reconstruido recientemente dos capillas católicas y dos «salas de silencio», diseñadas con la ayuda de una asociación interreligiosa y destinadas a ser un espacio para el uso de las minorías religiosas. Por lo tanto, en relación con el espacio, el hospital podría parecer menos reticente a la presencia de la religión que la prisión, pero de nuevo las cosas son más complejas de lo que parecen. Según Teresa, la trabajadora del servicio religioso, los miembros de las minorías religiosas casi no utilizan las salas de silencio y solo se abren para el personal médico y de enfermería que va allí a meditar en los ratos libres.
Estos ejemplos nos permiten ilustrar la imposibilidad de encontrar un modelo unificado, coherente y congruente para explicar el lugar que ocupa y el rol que desempeña la religión en las instituciones públicas en la España contemporánea (Astor 2014). Todo ello evidencia, por consiguiente, la idoneidad de privilegiar una metodología cualitativa que, a través de un procedimiento inductivo, permita captar la complejidad, contradicciones y matices de la realidad estudiada. Por este motivo, y con la voluntad de hacer viable la comparación entre ambas instituciones, este artículo toma como objeto de estudio los discursos y las actuaciones en torno a las cuestiones religiosas de los profesionales vinculados a los centros hospitalarios y penitenciarios. El objetivo final es comprender cómo ambas instituciones se adaptan y responden a los cambios recientes en el paisaje religioso. El hecho de centrar la atención en los actores institucionales responde a la convicción de que, como indican Powell y Colyvas (2008), el estudio de las instituciones y sus cambios requiere, necesariamente, comprender cómo los actores mantienen o transforman las fuerzas institucionales que guían sus prácticas cotidianas. Las 43 entrevistas realizadas a autoridades políticas, técnicos y otros trabajadores de prisiones y hospitales en el marco del proyecto de investigación "GEDIVER-IN. La gestión de la diversidad religiosa en hospitales y prisiones en España» son el material empírico principal utilizado para analizar cómo los actores conciben, interpretan y justifican sus acciones en torno a la religión y a la diversidad religiosa en estas dos instituciones. Sin embargo, con el fin de contextualizar los discursos, la información de las entrevistas a los actores institucionales se ha complementado con 58 entrevistas a actores religiosos y otros interlocutores ${ }^{2}$, y con el análisis documental y el trabajo etnográfico de observación realizado de forma intermitente entre 2012 y 2014 en 8 hospitales y 8 prisiones en Andalucía y Cataluña ${ }^{3}$. La observación se desarrolló, principalmente, en tres ámbitos distintos: a) actividades religiosas y no religiosas llevadas a cabo por los grupos religiosos y espirituales en prisiones y hospitales; b) salas de culto y observación en otros lugares comunes como bibliotecas o salas de espera; y c) actividades relacionadas con las terapias alternativas. Todas las observaciones se registraron en un diario de campo y, en los casos en que fue posible, se recogió también información visual.

2 Se han realizado 46 entrevistas a actores religiosos de 7 confesiones distintas, 6 a voluntarios o trabajadores de ONG de carácter religioso que intervienen en prisiones y/o hospitales y 6 a otros actores del campo.

3 Por cuestiones de espacio, en este artículo no ahondaremos en las diferencias entre ambos territorios. Cabe decir que ambas comunidades muestran un grado similar de diversidad religiosa pero difieren en el nivel de adhesión formal a la Iglesia católica. 


\section{SECULARIZACIÓN Y DESCONFESIONALIZACIÓN: ¿QUÉ ROL PARA LA RELIGIÓN EN LAS PRISIONES Y HOSPITALES CONTEMPORÁNEOS?}

España es un país que se enfrenta a un proceso de desmonopolización religiosa iniciado con la transición democrática. Hasta esa fecha, la Iglesia católica ocupaba una posición dominante y exclusiva en la mayoría de ámbitos sociales, como la educación, la salud o las instituciones penitenciarias. El proceso democrático debilitó su hegemonía y la Constitución definió el país como aconfesional, rechazando la idea de una religión de Estado pero atribuyendo un estatus especial a la Iglesia católica. Los acuerdos concordatarios entre el Estado Vaticano y el gobierno español firmados poco después de la aprobación de la Constitución Española de 1978 regulan, aún hoy, el papel de la Iglesia católica en el país. Posteriormente, se firmaron otros acuerdos específicos para desarrollar su papel en contextos concretos, como el Acuerdo sobre Asistencia Religiosa Católica en Centros Hospitalarios Públicos de 20 de diciembre de 1985 o el Acuerdo sobre Asistencia Religiosa Católica en los Establecimientos Penitenciarios de 24 de noviembre de 1993. Algunas de las cuestiones más importantes que regulan son el número de capellanes católicos con el que debe contar cada prisión u hospital de acuerdo con el número de presos/camas, y el coste de la asistencia católica -incluidos los sueldos de los capellanes- que el Estado español debe cubrir. A nivel legal, la diferencia entre prisiones y hospitales en esta materia es mínima y se limita al hecho de que, mientras para los hospitales queda regulada de manera más explícita la existencia de una capilla católica y el deber de proporcionar a los capellanes una oficina y un espacio para pernoctar, en el caso de las prisiones la regulación es menos estricta y se amolda a las condiciones específicas de los centros.

La separación política entre el Estado y la Iglesia católica en España fue acompañada de una caída drástica en la participación religiosa tradicional y en las tasas de identificación religiosa (Pérez-Agote 2012). La democracia trajo consigo la idea de modernidad secular, caracterizada por una concepción de la religión como fenómeno residual de un pasado en extinción (Estruch 1996). En este contexto, las «elites seculares» (tomando prestado el término propuesto por Peter Berger en 1999) ganaron protagonismo y visibilidad en la esfera pública (Díaz-Salazar 1990; 2007), y la histórica batalla entre laicos y clericales perdió parte de su virulencia. Hoy en día la semántica de la oposición entre lo religioso y lo secular ha cambiado notablemente, pero sigue sin existir consenso sobre el lugar que la religión -y en especial la Iglesia católica- debe ocupar en la sociedad en general y en las instituciones públicas en particular. La investigación cualitativa realizada en estos dos ámbitos institucionales (cárceles y hospitales) nos ha permitido detectar la existencia de una amplia gama de puntos de vista al respecto.

En este sentido, Xavier, un alto funcionario del Servei Català de la Salut, insiste en repetir que "la asistencia religiosa no entra en la cartera de servicios de la sanidad pública» cada vez que en la entrevista le preguntamos sobre la provisión de la asistencia religiosa. Para él, esto depende de la voluntad y autonomía de los centros. Xavier se muestra convencido de que la religión no tiene ni debería tener nada que ver con la asistencia sanitaria. Su punto de vista no es una excepción en los hospitales españoles, sino todo lo contrario.

Cuando le preguntamos a Martín -intensivista en Andalucía y presidente del comité de bioética del hospital donde trabaja- sobre el papel de la religión y las cuestiones que se plantean en materia religiosa en el hospital, nos responde que «esto es un Estado aconfesional y si un ciudadano pide que se le ponga un ladrillo en los pies de su familiar que va a fallecer, se le pone un ladrillo. ¿Qué más me da a mí un ladrillo?»; y añade «pero probablemente de lo que estamos hablando es de los testigos de Jehová, eso es de lo que estamos hablando». Martín se muestra reacio a considerar que, más allá de la cuestión de los testigos de Jehová y los protocolos elaborados en esta área, tenga sentido hablar o discutir sobre el papel de la religión en los centros hospitalarios. Es más, cuando le preguntamos sobre los retos existentes en materia religiosa, Martín responde de entrada: «te has pegado un buen palizón [refiriéndose al trayecto de la investigadora hasta el hospital] para algo que está colgado en Internet [los documentos relativos a las cuestiones bioéticas y testigos de Jehová]». Él considera que, con la excepción ya mencionada de los testigos de Jehová, no hay conflictos ni demandas desatendidas, y que la existencia de un marco jurídico aconfesional es condición suficiente para garantizar la neutralidad y secularidad de los centros hospitalarios. Como Martín, la mayoría de profesionales del ámbito sanitario entrevistados perciben la religión como un asunto completamente privado que pertenece a la esfera de la intimidad del paciente y donde el hospital no debe intervenir.

Solo esporádicamente se concede a la religión un papel en el marco hospitalario. Suele ser allá donde la medicina ya no tiene respuesta, en las unidades de cuidados paliativos, donde se otorga un mayor reconocimiento al papel que la religión puede jugar en el bienestar de los pacientes. Ahora bien, en el resto de momentos de la vida cotidiana de un hospital, la religión es percibida como ausente y se celebra que así sea. Los regímenes de conocimiento religioso y biomédico se consideran mutuamente excluyentes, e incluso, competidores, a la hora de dar respuestas a situaciones de enfermedad.

La situación no es la misma en las instituciones penitenciarias, donde la religión no es percibida en una 
relación de oposición sino de complementariedad con el objetivo de la prisión (García Martínez 2000). Es decir, se considera que la religión puede contribuir a fomentar la rehabilitación del interno $\mathrm{y}$, sobre todo, a mantener el orden en el centro penitenciario. En este sentido se expresa María, funcionaria del Ministerio de Justicia, quien afirma que la religión tiene mucha relevancia en el contexto de la prisión porque se encuentra en consonancia con la filosofía de la rehabilitación imperante hoy en día en esta institución. La religión es concebida como un recurso personal que los internos pueden utilizar para dar sentido a su situación, construir un relato significativo y, a la vez, proyectar un futuro para cuando obtengan la libertad (Becci 2011; García Martínez 2000; 2007; Restrepo y Moreno 2010). Con este cometido, las personas reclusas pueden beneficiarse del asesoramiento y consejo personal de los voluntarios y ministros religiosos y participar en actividades y celebraciones religiosas. Ahora bien, la religión es también percibida como un recurso social clave en el marco de escasez, exclusión social y vulnerabilidad del entorno carcelario. Los grupos religiosos desarrollan un papel crucial en la oferta de apoyo social y en la creación de mecanismos para favorecer la futura reinserción. Es más, parte de su legitimidad en prisión deriva del hecho de que contribuyen en buena medida a paliar las carencias y limitaciones del estado del bienestar español. Irene Becci (2011) destaca esta misma cuestión para el caso italiano y lo diferencia de lo que sucede en los centros penitenciarios de Suiza, donde la función del agente pastoral de prisiones se limita, de forma exclusiva, a las cuestiones espirituales. Más allá del ámbito penitenciario, Xabier Itçaina (2014) remarca que, tanto en Italia como en España, la acción social es una fuente clave de legitimidad social para la Iglesia católica.

La mayor valoración de la religión en el marco penitenciario también se explica por su función en el mantenimiento del orden social. En este sentido, Mercedes, una funcionaria de alto rango en la administración penitenciaria, nos explicaba que:

«Cualquier funcionario que trabaje en la institución penitenciaria sabe que cuanto más satisfechos estén los internos, que son los usuarios del sistema, en términos globales, menos conflictos le van a dar a él [...]. Luego, si el elemento de la asistencia religiosa ayuda a mantener el clima social adecuado, más nos vale a los funcionarios que trabajamos procurar que eso funcione bien, [...] la inteligencia lleva a pensar que si eso funciona bien, bueno, va a funcionar mejor el clima de la prisión y esa es una meta a la que uno aspira cada día cuando se pone el uniforme y entra a trabajar en una prisión».

Favorecer el rol de la religión en la prisión es una cuestión pragmática que forma parte del llamado «sentido común» burocrático de la institución. Como expone Adelantado (1992: 85), la paz institucional es el principal objetivo de la gestión carcelaria y contener el conflicto es prioritario «tanto políticamente como condición necesaria para la reeducación», y si los profesionales perciben que la religión puede ayudar en este cometido, facilitarán que así sea.

El análisis de los discursos de los profesionales muestra que existe una diferencia sustancial entre el papel atribuido a la religión en los hospitales y las cárceles. Los profesionales médicos, sobre todo aquellos que ocupan cargos de mayor responsabilidad, ven la religión en términos de incompatibilidad, mientras que la lógica de la complementariedad es la que predomina en la cárcel. Esta diferente consideración del rol de la religión en hospitales y prisiones la confirma, también, el análisis del discurso de los actores católicos.

En términos generales, los sacerdotes que trabajan en las instituciones sanitarias se sienten menos valorados y más cuestionados que sus homólogos en las prisiones. En su opinión, esto es especialmente evidente a raíz de la normativa de protección de datos aprobada en 1999 y la de autonomía del paciente de 2002, que indirectamente les impide obtener información de los pacientes, acceder a todas las habitaciones o entrar en todos los espacios de los edificios. Algunos sacerdotes católicos -y también pastores evangélicos- dicen sentirse discriminados por la «ideología laicista» que, según ellos, impera entre parte del personal de los centros. En este sentido, Alberto, delegado de pastoral hospitalaria, se quejaba en la entrevista de que el trabajo pastoral en el ámbito hospitalario

«Es una tarea difícil porque hay personal muy mal educado que discrimina y no tiene en cuenta los derechos humanos [...] Hay sacerdotes que no han podido aguantar más de 3 meses por las presiones» y añade "a mí, quien me diga que es una tontería que un enfermo baje a misa es que no lo entiende. Quizá para esta persona es una tontería, pero para el enfermo puede ser fundamental porque puede dar sentido a su vida».

Ahora bien, la casuística es amplia y, en general, el análisis muestra que la Iglesia católica detenta todavía una posición dominante tanto en el campo penitenciario como en el hospitalario. No se trata solo del hecho de que el sistema legal garantice la presencia estable de la Iglesia católica -en términos de recursos y facilidades- en estas instituciones, sino que las dinámicas institucionales forjadas históricamente y las sinergias del personal refuerzan esta situación. Nos referimos a la existencia de rutinas fuertemente entroncadas en el funcionamiento de estas instituciones que generalmente permanecen invisibles y naturalizadas a ojos de los trabajadores. Es lo que, en otra ocasión, y partiendo del concepto de nacionalismo banal acuñado por Michael Billig (1995), identificamos con la noción de «catolicismo banal» (Griera y Clot, 2015).

Prueba de ello es la celebración de festividades religiosas católicas, como Navidades O La Merced patrona de instituciones penitenciarias-, el indulto de presos durante Semana Santa a petición de las cofradías católicas y el acceso de capellanes a áreas restringidas, como las unidades de primer grado 0 aislamiento en 
algunos de los centros penitenciarios. Todos estos beneficios o ventajas son percibidos habitualmente como triviales por el personal de los centros, pero su rol es crucial a la hora de reforzar la hegemonía de la Iglesia en estos espacios. Ilustrativo de ello es el comentario de Ana, jefa de atención al usuario de un hospital comarcal, que afirmaba que «el cura tiene la costumbre de pasar por todas las habitaciones [aún sabiendo que está prohibido por ley] pero es un santo varón aquel hombre... Y, a ver, ayuda espiritual la da, y la da a todo el mundo sean moros, chinos...». La familiaridad, confianza y «normalidad percibida» (Goffman 1971) por parte del personal hacia la Iglesia católica es, en última instancia, la mayor fuente de poder del catolicismo en el seno de las instituciones públicas.

De modo similar, Sara, un cargo directivo de una prisión, nos respondía lo siguiente cuando le preguntamos por la presencia de símbolos religiosos en la instalación: «no hay símbolos, lo único que en este salón multiusos sí es verdad que hay una Virgen, pero no tanto por el símbolo religioso en sí, sino porque la tradición queda de la prisión antigua, que tiene muchísimos años esa talla y está ahí». Al vaciar de contenido religioso todos estos elementos y reformularlos en términos de tradición, el personal de las instituciones no hace sino integrarlos de manera todavía más sutil, agrandando aún más las distancias con la situación en que se encuentran las minorías religiosas.

\section{¿Desmonopolización de LAS INSTITUCIONES? LA DIVERSIDAD RELIGIOSA EN HOSPITALES Y PRISIONES}

La Ley de Libertad Religiosa 7/1980 sentó las bases jurídicas para el reconocimiento legal de las confesiones minoritarias, así como la garantía de la libertad religiosa y de conciencia de todos los ciudadanos. En el año 1992 los Acuerdos de Cooperación entre el Estado español y las confesiones musulmana, judía y protestante otorgaron a estas comunidades nuevos derechos y mayor reconocimiento en materia de libertad religiosa (Moreno Antón 2006). De manera específica, los Acuerdos de Cooperación reconocen el derecho a la asistencia religiosa en las instituciones públicas afirmando que: «Se garantiza el ejercicio del derecho a la asistencia religiosa de los internados en centros o establecimientos penitenciarios, hospitalarios, asistenciales u otros análogos del sector público» (artículo 9). Sin embargo, y a pesar de la importancia simbólica del marco legal, su implementación real fue muy limitada inicialmente ${ }^{4}$.

4 Asimismo, es importante enfatizar que el derecho de libertad religiosa en cárceles (Gallizo 2009) y hospitales (Molano 1987; Fernández Arruty 1996) es un derecho de todos los ciudadanos ampliamente recogido en la normativa internacional.
Ahora bien, en la década de los años 2000 el número de personas nacidas en el extranjero residentes en España creció sustancialmente, pasando de 1,5 millones a más de 6,5 millones (Arango 2009), lo que favoreció un crecimiento sustancial de las minorías religiosas. Este cambio ha tenido un claro impacto en las cárceles, donde más de un $24 \%$ de la población (Gallego et al. 2010) se declara creyente de una confesión distinta a la católica, mientras en el conjunto de la población española apenas supera el $2 \%$ (Centro de Investigaciones Sociológicas 2013). Asimismo, la encuesta realizada por Gallego et al. (2010) mostró que el 39,1\% de los presos se identifican como católicos no practicantes, el 23,1 \% como católicos practicantes, el 12,2\% como protestantes, el 5,7 \% como musulmanes, el 6,2\%, como miembros de otras religiones y el $13,8 \%$ como ateos o agnósticos. En relación con los hospitales, aunque no se dispone de datos sobre la afiliación religiosa de la población usuaria, es de suponer que el impacto actual sea menor que en las prisiones dada la media de edad de la población inmigrante y la menor duración de los ingresos. Sin embargo, la mayoría de entrevistados reconocen que la diversidad religiosa ha ganado visibilidad a raíz de la inmigración.

En ambas instituciones todos los profesionales reconocen la importancia de garantizar la libertad religiosa. No obstante, si bien a nivel de principios abstractos todos los entrevistados enfatizan la necesidad de respetar los derechos de los usuarios y de acomodar la diversidad religiosa, la situación cambia cuando el discurso versa sobre cuestiones concretas y específicas que afectan al funcionamiento cotidiano de la institución. Con frecuencia las demandas de las minorías religiosas generan malestar -e incluso en ocasiones irritación- entre el personal. Esto ocurre especialmente cuando aquellas interfieren de manera más evidente en los objetivos y la cultura organizacional de la institución.

Como ya hemos mencionado, el mantenimiento del orden y de las rutinas institucionales es una de las cuestiones principales en una cárcel. La celebración del Ramadán -que según cifras del Departament de Justícia de la Generalitat de Catalunya en algunas prisiones es seguido por más del $40 \%$ de los internos- genera un reto en términos organizativos y rompe las dinámicas cotidianas del centro. En este sentido, un técnico de la administración apuntaba que "seguramente [el malestar respecto a la celebración del Ramadán] tiene más relación con la preocupación que hay en las prisiones de seguir con la rutina habitual; cualquier cosa que implique salir de la rutina habitual es visto como una molestia».

Ahora bien, las cuestiones relacionadas con la ruptura de las rutinas diarias no lo explican todo. De hecho, en una parte importante de las prisiones españolas la oración colectiva del viernes no está permitida o cuenta con restricciones. Si bien el derecho no se niega de manera 
abierta, se mencionan otras razones que de modo indirecto impiden la celebración: la falta de imames «adecuados», la escasez o ausencia de espacios para dichas celebraciones o la escasa demanda por parte de los propios internos. Óscar, un técnico de la administración, mostraba de forma clara la lógica que se esconde tras estos discursos y prácticas:

«Piensa que coincide también con el tema del $11 \mathrm{M}$ cuando empieza todo esto. Entonces siempre se ha mirado el tema Ramadán, el tema de los islámicos como un poco, como con cuidado, recelosos, pero a la vez te diría que para ser prisiones con una actitud bastante abierta. Porque bueno, hay siempre control, no te voy a decir que no, siempre se está mirando un poco diferente que si son testigos de Jehová que mientras los dejan hacer... Con ellos [los musulmanes] siempre hay un poco de, pues que no te estén colando un discurso integrista».

Si en el caso de las prisiones son las cuestiones vinculadas con las demandas por parte de musulmanes de celebrar el Ramadán lo que genera mayores reticencias, en los hospitales lo es el rechazo por parte de pacientes testigos de Jehová de las transfusiones de sangre. En los últimos años, los hospitales españoles han trabajado de manera conjunta con diferentes órganos de la administración de salud del gobierno central y de las autonomías para elaborar protocolos que faciliten el respeto a los derechos de estos pacientes y, al mismo tiempo, protejan al personal sanitario ante situaciones de difícil resolución. Según nuestros entrevistados, la mayoría de controversias relacionadas con este asunto se han reducido de manera significativa, pero el tema todavía emerge en todas y cada una de las entrevistas realizadas en el ámbito sanitario. Según nuestros interlocutores, todavía hay facultativos que se resisten a atender a pacientes testigos de Jehová o que optan por la objeción de conciencia antes de participar en una intervención a un testigo. Como nos contaba
Marta, una oncóloga que preside el comité de bioética del hospital en el que trabaja: "[que su religión sea más importante que morirse] es algo que les cuesta mucho aceptar a los profesionales. Los profesionales se arman un lío como una casa porque piensan que si atienden a esas demandas, luego les demandarán». El rechazo de las transfusiones de sangre por parte de los testigos de Jehová es percibido como algo que atenta contra el «sentido común» institucional y que va en contra de, u obstaculiza, el objetivo último de la medicina de curar el cuerpo.

Como se ha evidenciado hasta ahora, las respuestas dadas a las demandas religiosas se encuentran condicionadas por las normas, principios y aspectos organizativos de cada institución. Esto se hace evidente también en la forma como se gestiona la atención religiosa para pacientes y reclusos pertenecientes a las minorías religiosas. Así, el modelo de provisión varía mucho entre hospitales y prisiones. Las características de las prisiones, y en especial el hecho de tratarse de instituciones totales, obligan a que la atención religiosa sea organizada y gestionada por parte de la propia institución. Los protocolos de acceso para los ministros de culto se encuentran altamente estandarizados y los procedimientos de acreditación y acceso son muy uniformes. Los representantes religiosos solo pueden acceder a las prisiones si al menos un interno así lo ha solicitado y están acreditados por el Ministerio de Justicia, o el Departament de Justícia en el caso de las prisiones catalanas (Martínez-Ariño et al. 2015).

Como indican los datos referentes a las personas autorizadas para ofrecer asistencia religiosa en las cárceles españolas, el servicio se ha diversificado de manera notable. También ocurre lo mismo en el caso de los hospitales, donde, cada vez más, representantes de las minorías religiosas

Tabla 1.

Actores religiosos acreditados como voluntarios de prisiones y su distribución en las cárceles españolas.

\begin{tabular}{lccc}
\hline & Actores religiosos con autorización & $\begin{array}{c}\text { Prisiones con presencia de } \\
\text { confesiones religiosas (80 prisiones } \\
\text { en total) }\end{array}$ & $\begin{array}{c}\text { \% de prisiones que tienen presencia } \\
\text { de las confesiones religiosas }\end{array}$ \\
\hline Catolicismo & 318 & 80 & 100 \\
\hline Islam & 24 & 23 & 28,75 \\
\hline Protestantismo & 268 & 50 & 62,5 \\
\hline $\begin{array}{l}\text { Iglesia Adventista del Séptimo } \\
\text { Día }\end{array}$ & 15 & 7 & 8,75 \\
\hline lglesia Ortodoxa & 21 & 19 & 23,75 \\
\hline Testigos de Jehová & 245 & 42 & 52,5 \\
\hline Judaísmo & 1 & 1 & 1,25 \\
\hline Budismo & 1 & 1 & 1,25 \\
\hline
\end{tabular}

Fuente: Elaboración propia, a partir de la información del Ministerio de Justicia y del Departament de Justícia de la Generalitat (2011). 
acceden para ofrecer acompañamiento religioso, si bien esta presencia resulta prácticamente invisible a nivel institucional. Más allá de contadas excepciones, los hospitales no disponen de procedimientos formales para ofrecer asistencia religiosa a los pacientes de las minorías religiosas y predomina la informalidad. La responsabilidad recae mayoritariamente en las familias o comunidades religiosas, quienes se encargan de organizar la atención a la vida espiritual de los pacientes y sufragar sus necesidades. llustrativo de esto es el caso de una comunidad judía de Andalucía, que se organiza para suplir esos vacíos asistenciales, como nos señalaba su rabino:

«hay un grupo de señoras organizadas para dar facilidades al enfermo que esté en el hospital y a la familia del enfermo, ¿ok? Principalmente con el tema alimenticio (sic), porque nosotros tenemos una dieta especial que es el kosher, y los hospitales no tienen catering kosher, ¿ok? Entonces para facilitar el alimento kosher a todas las personas que están en los hospitales, este grupo de señoras se reparte los trabajos y cada día una prepara un tipo de alimento y se lo acerca al hospital».

El caso de los hospitales es especialmente complejo, dada su gestión descentralizada y la autonomía de que gozan. Ahora bien, excepcionalmente, en algunos hospitales pudimos detectar el desarrollo de prácticas más inclusivas: la construcción de una capilla multiconfesional, la institucionalización y acreditación del servicio de asistencia pastoral de alguna de las minorías religiosas o la promoción de protocolos de colaboración entre las minorías religiosas y la institución, en particular en el caso de los testigos de Jehová. Aunque cada caso es singular y tiene su propia genealogía, la mayoría de ellos comparten un rasgo común: la acción de personas que individualmente actúan de manera altruista en nombre de los derechos de las minorías y que ellas mismas son miembros activos de una confesión religiosa. Es el caso de una enfermera evangélica que logró que su hospital incluyera en el folleto de ingreso el teléfono de contacto de diferentes comunidades religiosas diferentes de la católica o el de una trabajadora católica que impulsó la construcción de un espacio de silencio. En términos comparativos, los hospitales son más permeables que las prisiones a los «emprendedores institucionales». Ahora bien, a nivel general, mientras la administración penitenciaria actúa proactivamente para favorecer los derechos de las minorías religiosas, la lógica de los centros hospitalarios es de carácter reactivo: si no hay una demanda explícita 0 un problema evidente, raramente el centro hospitalario dispondrá de una política institucionalizada en esta materia.

El rol de la Iglesia católica en este escenario es complejo. En algunas instituciones, y especialmente en los hospitales, los profesionales entrevistados afirman que el capellán católico actúa como «bróker» (Beckford y GilliatRay 1998) entre las minorías religiosas y el centro, ya sea contactando personalmente con un ministro de culto de una minoría cuando un paciente lo reclama, o mediando entre las necesidades de los creyentes y la institución. Esta no es una opción generalizada y hay sacerdotes que no están interesados ni dispuestos a realizar esta función. Sin embargo, lo que sí es evidente es que en ningún caso encontramos un rechazo generalizado de la Iglesia católica hacia las minorías. Así, es más clara su resistencia hacia las autoridades políticas que, en ocasiones, son percibidas como propulsoras de una política «laicizadora», que no hacia las otras confesiones religiosas. Son las medidas secularizadoras del Estado, como la limitación de los espacios de uso exclusivo para el culto en el marco de las instituciones, y no la presencia de las minorías, las que son vividas como un ataque y contestadas con una resistencia explícita. La lógica de oposición entre las autoridades públicas seculares y la Iglesia católica es mucho más evidente que entre esta última y las minorías.

\section{¿MÁs ALLÁ de LA RELIGIÓN? LA PRESENCIA EMERGENTE DE ESPIRITUALIDADES HOLÍSTICAS EN HOSPITALES Y PRISIONES}

Meditaciones zen, sesiones de yoga, iniciaciones al reiki, cursos de astrología, constelaciones familiares 0 seminarios de relajación para profesionales son algunas de las muchas y variadas expresiones de la llamada nueva espiritualidad que también hemos encontrado en las prisiones y hospitales españoles. Si bien inicialmente en muchas ocasiones pasan desapercibidas a primera vista, una mirada más atenta revela la existencia en dichas instituciones públicas de un entramado de prácticas y técnicas holísticas que añade complejidad al mapa dibujado por las distintas confesiones religiosas.

De manera similar a las dinámicas socio-religiosas existentes en la actualidad en el contexto español (Cornejo 2012; Prat 2012; Griera y Urgell 2002), desde finales de los años noventa aproximadamente, junto con el gradual aumento de la diversidad religiosa, constatamos una presencia creciente de expresiones holísticas en los contextos penitenciario y hospitalario. Los portadores -en el sentido weberiano del término- de esta amalgama de técnicas y prácticas son, principalmente, trabajadores/as y educadores/as sociales así como algunos psicólogos y pedagogos en el caso de prisiones y, generalmente, personal de enfermería en el caso de hospitales. Se trata, en todo caso, de profesionales de medio rango que trabajan en la primera línea de estas instituciones. Procedentes de una clase media educada y siendo fundamentalmente mujeres -en consonancia con los patrones de la espiritualidad actual (Sointu y Woodhead 2008; Fedele y Knibbe 2013)-, la formación en estas técnicas holísticas surge de un interés personal y escapa 
de los círculos más institucionalizados de los diferentes grupos religiosos, tomando caminos variados y fuentes eclécticas. Dada la versatilidad de estas prácticas, situadas en la frontera difusa entre educación, salud, ciencia y espiritualidad, su aplicación en el contexto institucional penitenciario y hospitalario se funde con la actividad formal de dichas instituciones, creando sinergias y complicidades entre los profesionales que comparten un mismo bagaje y sensibilidad por estos temas.

Estos nuevos lenguajes y prácticas espiritualterapéuticas penetran en ambas instituciones a través de un proceso bottom-up, es decir, a partir del interés e ideas de los propios profesionales. Este es el caso, por ejemplo, de los cursos de yoga o reiki en las prisiones. Tal y como nos contaban Manuel y Sandra, un educador y una monitora de un centro penitenciario, la propuesta de hacer yoga surgió del propio equipo de educadores. También es el caso del reiki en otra prisión, donde Marta nos puntualizaba que fueron ellas mismas, las educadoras sociales, quienes adoptaron la iniciativa de impulsar un curso. Del mismo modo, si bien en menor medida, también encontramos evidencia de esto en los centros hospitalarios. Silvia, una enfermera entrevistada nos contaba que «hay alguna enfermera a nivel individual que ha hecho algún postgrado en terapias alternativas y que, individualmente, como experiencia propia, ofrece esto para sumar a la atención». En general los relatos de los portadores siguen un mismo patrón: la voluntad de impulsar estas actividades nace a raíz del impacto positivo de estas prácticas en su vida particular y la creencia de que también puede ser de gran ayuda para usuarios y colegas (Griera y Clot, 2015b).

Tanto en prisiones como en hospitales, por consiguiente, la introducción de estas actividades no se enmarca en términos espirituales o religiosos, sino que inicialmente se presenta acompañada de una justificación educativa -en prisiones-, como mejora de la calidad de la atención integral al paciente -en hospitales- o para el bienestar del personal. En ambos contextos, estas actividades, más que institucionalizarse, acaban adoptando la forma de voluntariado, como actividades de ocio educativo o actividades complementarias. Así Ramón, un educador social de una prisión, explicaba: «los internos tienen tiempo libre los fines de semana y actualmente no tenemos presupuesto para actividades especiales. Por tanto, si puedo llevar voluntarios que enseñen yoga, reiki, zen o cosas similares de forma gratuita para los internos, los directores no preguntan». La cuestión clave para entender la postura de la dirección en ambas instituciones respecto a la presencia legítima de estas técnicas holísticas no es tanto el reconocimiento de los efectos beneficiosos que puedan tener, sino el hecho de que no interfieran en el funcionamiento ordinario de dichas instituciones. Dicho de otro modo, al no entrar en contradicción con cuestiones de seguridad -en prisiones- ni criterios asistenciales -en hospitales-, estas actividades cuentan con la aprobación 0 , al menos, la no oposición formal de las direcciones de los centros. Ahora bien, raramente encontraremos que sean aceptadas e incorporadas de forma explícita.

En su conjunto, todo este abanico de técnicas y prácticas holísticas, que abarcan desde el yoga hasta las constelaciones familiares, configura en las instituciones públicas españolas lo que Becci y Knobel (2014) han identificado en las prisiones alemanas como «grey zones». Se trata de expresiones que se sitúan al margen de las afiliaciones religiosas formales y de los servicios religiosos regulares. Todas estas actividades holísticas, por tanto, no se consideran religiosas ni a nivel formal por parte de la dirección de los centros ni a nivel informal por parte de los profesionales que las propulsan. Una frontera clara se establece entre aquello que es religioso y lo que pueda tener, eventualmente según los entrevistados, una dimensión espiritual. En palabras de Ramón: «esto es diferente de la religión... es una experiencia holística que ayuda a los internos a elevar su nivel de conciencia». No obstante, muchos de estos cursos tienen contenidos explícitos de religiones orientales y en algunos casos son conducidos por miembros de las propias minorías religiosas -sobre todo Brahma Kumaris y budistas- que están registrados como tales en el Ministerio de Justicia de España. Por tanto, y a pesar de los diferentes canales institucionales por los que discurren las prácticas religiosas tradicionales y las prácticas holísticas, las fronteras se presentan difusas.

Algunas de estas actividades están directamente dirigidas a los profesionales, sobre todo en los hospitales. En este sentido Teresa, la trabajadora del servicio religioso de un hospital católico, comentaba que la actividad más exitosa del equipo del servicio religioso era, precisamente, el encuentro bianual de relajación y meditación. Este día de la meditación, organizado por un hermano de la orden del hospital con experiencia en religiones orientales y conocido por promover una espiritualidad abierta, agrupa a más de cien profesionales de la salud y otros miembros del personal. Esta noción de espiritualidad abierta también está ganando terreno entre los profesionales de medicina paliativa, tal y como lo ilustra el Grupo de Trabajo sobre Espiritualidad de la Sociedad Española de Cuidados Paliativos (SECPAL), que trabaja para promover una noción amplia de espiritualidad en el contexto hospitalario. En las diferentes actividades que organiza (celebración de seminarios o publicación de informes sobre el tema) se distancia de manera explícita de perspectivas clásicas sobre la religión. Esta estrategia, que apuesta por la complementariedad entre espiritualidad y medicina paliativa, le permite alcanzar una cierta legitimidad en un contexto en el que formas más tradicionales de religiosidad y referencias a la Iglesia católica han perdido buena parte de su prestigio. En su conjunto, la realidad empírica tanto 
en contextos penitenciarios como hospitalarios nos lleva a subrayar la presencia silenciosa aunque in crescendo de las prácticas holísticas.

\section{Conclusiones}

El mapa religioso en España ha cambiado profundamente en los últimos años con la consolidación del proceso de secularización, el crecimiento de las minorías religiosas y la emergencia de nuevas formas de espiritualidad. Todos estos elementos se reflejan en el papel multifacético y ambiguo que la religión desempeña hoy en día en el marco de las instituciones públicas. El análisis de los discursos de los actores institucionales y la variabilidad de sus prácticas muestra la dificultad de identificar un modelo coherente que nos permita explicar de forma univoca el rol de la religión en las instituciones penitenciarias y hospitalarias. En este sentido, la investigación nos muestra que, heurísticamente, es más pertinente explicar las transformaciones en el papel de la religión en las instituciones públicas contemporáneas en términos de tensión y negociación entre fuerzas dicotómicas que actúan de manera paralela: lo secular/religioso; lo monoconfesional/multiconfesional y lo espiritual/religioso. Estas tres lógicas de tensión se solapan entre sí y la morfología de cada una de ellas cambia en los contextos institucionales particulares.

Por un lado, el estudio pone de manifiesto que, lo que Lori Beaman y Winnifred Sullivan (2013) caracterizan como el mantra del estado secular, ha tenido más éxito en hospitales que en prisiones. Los profesionales sanitarios ilustran lo que Peter Berger (1999) llama las «elites seculares» y perciben la religión en clara oposición con el objetivo del hospital. No es este el caso en las prisiones, donde la religión es concebida como una fuerza aliada y en complementariedad con los objetivos de la institución. Además, el rol de las instituciones religiosas a la hora de paliar las carencias de la institución en términos sociales les atribuye aún mayor legitimidad en este contexto. Ahora bien, y más allá de las declaraciones formales de principios de los profesionales, el análisis meticuloso del discurso de las entrevistas y la observación etnográfica revelan la existencia de un «catolicismo banal» que se hace presente en las rutinas de la institución. Nos referimos a cuestiones como la familiaridad y confianza en el trato con los actores católicos -especialmente evidente cuando lo comparamos con el que reciben las minorías-, la existencia de un entramado de prácticas que perviven en el tiempo, como los indultos en Semana Santa, o las celebraciones católicas, entre otras muchas. El «catolicismo banal» naturaliza, desproblematiza y convierte en invisible la hegemonía católica en el marco de las prisiones y los hospitales a la vez que contribuye a reforzar la desigualdad estructural entre minorías religiosas e Iglesia católica.
Sin embargo, es importante puntualizar que estas instituciones no son monolíticas y nos encontramos, también, con la existencia de fuerzas secularizadoras de índole diversa que ponen en entredicho la preeminencia católica y cuestionan, de forma directa o indirecta, el "catolicismo banal». Ya hemos mencionado el impacto de la normativa relativa a la protección de datos para los sacerdotes católicos que trabajan en los hospitales. Podríamos referirnos también a la desaparición de las capillas católicas en algunas cárceles 0 a la limitación de, y los debates entorno a, la posibilidad de los sacerdotes de participar en los comités de bioética de los hospitales y las juntas de tratamiento de los centros penitenciarios.

En relación con el impacto de la diversidad religiosa en estas dos instituciones, las entrevistas muestran que las instituciones penitenciarias tienen una política más proactiva hacia la diversidad religiosa que los hospitales. Sin embargo, el enfoque resultante es también más uniforme, constrictor y homogeneizante que el de los hospitales. La gestión de la diversidad religiosa dentro de una «institución total» (Goffman 1961), es decir, altamente cerrada y estructurada como la prisión, implica la necesidad de construir categorías, normas y procedimientos muy claros. Esto puede dar lugar a la imposición de una noción reificada de religión (Beckford 2009) que no tenga en cuenta las variaciones individuales o la diversidad intrarreligiosa y favorezca la aplicación de un enfoque más intervencionista (Bader 2007).

Por el contrario, en el caso de los hospitales, la ausencia de un enfoque global hacia la acomodación de la diversidad religiosa deja un espacio mayor para la acción individual y la variabilidad. La gestión de la diversidad religiosa no es percibida como una prioridad en el contexto hospitalario $y$, en general, las demandas de las comunidades religiosas permanecen invisibilizadas. El laissez faire obliga a que sea el paciente o su familia quien se responsabilice de la atención religiosa, poniéndose en contacto con su propio ministro de culto o voluntario religioso. Solo en aquellos casos en los que hay un conflicto, o una petición formal y expresa, los centros ponen en marcha dispositivos de atención. Ahora bien, el contexto del hospital favorece en mayor medida el surgimiento de "emprendedores institucionales», que se convierten en agentes clave en el fomento de nuevos enfoques hacia la diversidad religiosa en los hospitales.

La investigación también ha revelado la creciente existencia de una amalgama de prácticas que, a medio camino entre la religión, la ciencia y la salud, se identifican con una concepción difusa de espiritualidad. El entorno penitenciario es especialmente receptivo a la incorporación de estas actividades que, de la mano de los profesionales mismos, va adquiriendo protagonismo. La necesidad impertérrita de las cárceles de "llenar el tiempo» de los internos, junto con la existencia de voluntarios/as 
dispuestos a dedicar su tiempo a esta cuestión, dan mayor cabida a estas prácticas en un contexto de crisis y de reducción de programas formales. Detectamos, también, la emergencia de este tipo de prácticas en los centros hospitalarios, si bien de forma mucho más modesta y más dirigido a los propios profesionales que a los pacientes. En ambos contextos, la frontera entre religión y espiritualidad es, fundamentalmente, una distinción emic de los actores fundada en la percepción del desprestigio social de la religión y la valoración y popularización de una llamada «espiritualidad abierta» (Fedele and Knibee 2013; Cornejo 2012).

En su conjunto, constatamos la existencia de numerosos factores distintos que juegan un papel en la conformación del enfoque de las instituciones públicas hacia la religión y en el análisis es muy importante tener en cuenta que su impacto puede tener efectos contradictorios. En particular, hay dos grupos de factores que deben ser tenidos en cuenta si queremos explicar las diferencias y similitudes en la forma de abordar las cuestiones religiosas en hospitales y cárceles: por un lado, los factores endógenos -relacionados con las propias instituciones- y, por otro lado, los factores exógenos -relacionados con la dinámica general del contexto religioso-.

Los factores endógenos hacen referencia a lo que Ines Michalowski (2010) denomina la estructura de oportunidad de la institución. Así, tal y como se ha evidenciado, la particular naturaleza institucional y las características propias de hospitales y prisiones deben ser contempladas a fin de explicar cómo cada una de estas instituciones afronta las cuestiones religiosas. El objetivo final declarado de cada institución -curar el cuerpo en el caso de los hospitales y rehabilitar a las personas internas en el caso de las prisiones- junto con la cultura organizativa de cada una de ellas influye en los discursos en torno al hecho religioso y en la definición e implementación de prácticas y estrategias concretas para encajarlo con el funcionamiento cotidiano de los centros. Así, aquello que se considera apropiado en un contexto puede ser percibido como inadecuado en el otro y viceversa. Además, el hecho de que en prisiones la duración media de la estancia de una persona sea mucho mayor que en el caso de los hospitales también condiciona de forma significativa la aproximación de la institución a las cuestiones religiosas. Así, mientras la administración penitenciaria se considera la única responsable de satisfacer las demandas religiosas de la población interna y actúa en consecuencia, en general las administraciones de los centros hospitalarios no perciben como propia dicha responsabilidad, sino que la delegan en mayor medida a parientes y correligionarios. Asimismo, para entender las diferencias entre ambas instituciones también es fundamental tener en cuenta que, mientras los centros hospitalarios gozan de gran legitimidad social, los centros penitenciarios necesitan legitimar su presencia de forma reiterada ante los internos y la sociedad en general. En este sentido, determinadas cuestiones como la provisión de asistencia religiosa y la garantía de los derechos en esta materia pueden ser una fuente de legitimidad (Bowen et al. 2014). En cambio, en los centros hospitalarios, la presión por respetar los derechos en esta materia es secundario y el impacto de hacerlo (o no) sobre la legitimidad social de la institución es muy limitado.

Por otro lado, es necesario tener en cuenta las múltiples y complejas transformaciones que ha sufrido el contexto religioso en las últimas décadas (Cornejo 2012; Díez de Velasco 2010; Estruch et al. 2004) y que han potenciado la emergencia de nuevos retos para las instituciones públicas. En esta línea, es especialmente relevante mencionar, en primer lugar, la consolidación del proceso de secularización, tanto a nivel institucional como social, que pone en cuestión el monopolio de la Iglesia católica; $y$, en segundo lugar, el crecimiento y la mayor visibilidad de la diversidad religiosa a raíz del incremento de los flujos migratorios internacionales (Martínez-Ariño et al. 2011; Briones 2010; Lacomba 2001). A todo ello se suman las preocupaciones de seguridad con respecto a las poblaciones musulmanas, derivadas principalmente de los ataques del $11 \mathrm{M}$ en Madrid, y el espectacular impulso de las nuevas formas de espiritualidad que se expanden en paralelo a la popularización de las terapias holísticas y la expansión de las religiones orientales (Griera y Urgell 2002). Todas estas transformaciones se han producido en un lapso de tiempo relativamente corto y su impacto en las instituciones aún no ha cristalizado de manera consistente. Nos encontramos, más bien, con un panorama heterogéneo en constante cambio y lleno de sutilidades y matices que es necesario seguir explorando.

\section{ReferenCias BibliográficAs}

Adelantado, J. 1992. «Disciplina social y organización interna de la cárcel. Síntesis del estudio de una prisión catalana». Papers. Revista de Sociología 39: 77-100.

Arango, J. 2009. «Después del gran boom. La inmigración en la bisagra del cambio». Pp. 53-73 en La inmigración en tiempos de crisis. Anuario de la inmigración en España, editado por Eliseo Aja, Joaquín Arango y J. Oliver. Barcelona: Fundació CIDOB.

Astor, A. 2013. «Religious Governance and the Accommodation of Islam in Contemporary Spain». Journal of Ethnic and Migration Studies: 1-20.

Beaman, L. G. y Winnifred Fallers Sullivan. 2013. «Neighbo(u) rly Misreadings and Misconstruals: A Cross-border Conversation». Pp. 1-11 en Varieties of Religious Establishment, compilado por W. F. Sullivan y L. G. Beaman. London: Ashgate Publishing.

Becci, I. 2011. «Religion's Multiple Locations in Prison: Germany, Italy, Swiss». Archives des sciencies sociales des religions 153: 65-84.

Becci, I. y Brigitte Knobel. 2014. «La diversité religieuse en prison: entre modeles de regulation et emergence de zones grises 
(Suisse, Italie et Allemagne)». Pp. 109-121 en Quand le religieux fait conflit. Désaccords, négociations ou arrangements, compilado por A. S. Lamine y N. Luca. Paris: La Découverte.

Beckford, J. y Sophie Gilliat. 1998. Religion in prison: 'equal rites' in a multi-faith society. Cambridge: Cambridge University Press.

Beckford, J. Daniele Joly y Farhad Khosrokhavar. 2005. Muslims in prison: Challenge and change in Britain and France. London: Palgrave Macmillan.

Beckford, J. 2009. «Power and the Reification of "Religion" in Prisons». Comunicación presentada en la Conferencia Interrogating Religion, 17-19 abril, Ottawa.

Berger, P. 1999. "The desecularization of the World: A Global Overview». Pp. 1-18 en The Desecularization of the World. Resurgent Religion and World Politics, compilado por P. Berger. Washington, D.C: Ethics and Public Policy Center \& William B. Eerdmans Publishing Company.

Billig, M. 1995. Banal nationalism. London: Sage Publications Ltd.

Bowen, J. R., Christophe Bertossi, Jan Willem Duyvendak y Mona Lena Krook. 2014. European States and their Muslim Citizens. New York: Cambridge University Press.

Briones, R. et al. 2010. ¿Y tú (de) quién eres?: Minorías religiosas en Andalucía. Barcelona: Icaria.

Cadge, W. 2013. «Negotiating religious differences in secular organizations: The case of hospital chapels». Pp. 200-214 en Religion on the edge: De-centering and re-centering the sociology of religion, compilado por C. Bender, W. Cadge, P. Levitt y D. Smilde. New York: Oxford University Press.

Cornejo, M. 2012. «Religión y espiritualidad, ¿dos modelos enfrentados?. Trayectorias poscatólicas entre budistas Soka Gakkai». Revista Internacional de Sociología 70: 327-346. http://dx.doi.org/10.3989/ris.2010.09.08

Díaz-Salazar, R. 1990. «Política y Religión en la Espa-a Contemporánea». REIS: Revista Española de Investigaciones Sociológicas 52: 65-84.

Díaz-Salazar, R. 2007. Democracia laica y religión pública. Madrid: Taurus.

Díez de Velasco, F. 2010. «The Visibilization of Religious Minorities in Spain». Social Compass 57: 235-252. http://dx.doi. org/10.1177/0037768610362410

Estruch, J. 1996. Secularitzacio i pluralisme en la societat catalana d'avui. Barcelona: Institut d'Estudis Catalans.

Estruch, J., Joan Gómez, Mar Griera, y Agustí Iglesias. 2004. Les altres religions. Minories religioses a Catalunya. Barcelona: Mediterrània.

Fabretti, V. 2014. «Dealing with religious differences in Italian Prisons: relationships between institutions and communities from misrecognition to mutual trasformation». International Journal of Politics, Culture, and Society 1-15.

Fedele, A. y Kim Knibbe. 2013. Gender and Power in Contemporary Spirituality: Ethnographic Approaches. Oxford: Routledge.

Fernández Arruty, J. A. 1996. «La asistencia religiosa en los centros hospitalarios públicos en España». Dereito: Revista Xurídica da Universidade de Santiago de Compostela 5: 71-84.

Furseth, I. 2000. «Religious Diversity in Prisons and in the Military: The Rights of Muslim Immigrants in Norwegian State Institutions». International Journal on Multicultural Societies 2: 40-52.

Furseth, I. 2003. «Secularization and the role of religion in state institutions». Social compass 50: 191-202. http://dx.doi. org/10.1177/0037768603050002005

Furseth, I. y Lene Kühle. 2011. «Prison Chaplaincy from a Scandinavian perspective». Archives de sciences sociales des religions 153: 123-141. http://dx.doi.org/10.4000/assr.22784

Gallego, M., Pedro José Cabrera, Julián Carlos Ríos y José Luis Segovia. 2010. Andar $1 \mathrm{Km}$ en línea recta: La cárcel del siglo XXI que vive el preso. Madrid: Universidad Pontificia de Comillas.
Gallizo, M. 2009. «Los derechos fundamentales de los presos. Especial referencia al derecho de libertad religiosa». Anuario de derecho eclesiástico del Estado 25: 91-108.

García Martínez, J. 2008. «Funcionalidad psico-social de las creencias en prisión». Acciones e investigaciones sociales 25: 171-200.

García Martínez, J. 2000. Sociología del hecho religioso en prisión. Salamanca: Universidad Pontificia de Salamanca.

Goffman, E. 1961. Asylums: Essays on the social situation of mental patients and other inmates. New York: Anchor Books.

Goffman, E. 1971. Relations in public: Microstudies of the social order. London: Allen Lane.

Griera, M. y Ferran Urgell. 2002. Consumiendo religión. Nuevas formas de espiritualidad entre la población juvenil. Barcelona: Fundación "la Caixa".

Griera, M. y Anna Clot. 2015. «Banal is not trivial. Visibility, recognition and inequalities between religious groups in prison». Journal of Contemporary Religion 30(1): 23-37. http://dx.doi.org/10.1 080/13537903.2015.986974

Griera, M. y Anna Clot. 2015b "Doing Yoga Behind Bars: A sociological study of the Growth of Holistic Spirituality in Penitentiary Institutions". In Becci, I and Roy, O. (eds.) Religious Diversity in European Prisons: Challenges and Implications for Rehabilitation. The Netherlands: Springer.

Itçaina, X. 2014. "Catholicism, social economy and local welfare in times of crisis: comparing Spanish and Italian territories». EUI Working Papers 02, European University Institute, Florencia.

Joly, D. y James Beckford. 2006. «"Race” Relations and Discrimination in Prison: The Case of Muslims in France and Britain». Journal of Immigrant \& Refugee Studies 4: 1-30. http:// dx.doi.org/10.1300/J500v04n02_01

Lacomba Vázquez, J. 2001. El islam inmigrado. Transformaciones y adaptaciones de las prácticas culturales y religiosas. Madrid: Subdirección General de Museos Estatales.

Martínez-Ariño, J., Mar Griera, Gloria García-Romeral, y María Forteza, M. 2011. «Inmigración, diversidad religiosa y centros de culto en la ciudad de Barcelona». Migraciones (30):101-33.

Martínez-Ariño, M, Gloria García-Romeral, Gemma Ubasart, y Mar Griera. 2015. «Demonopolization and dislocation: (re-) negotiating the place and role of religion in Spanish prisons». Social Compass 62(1): 3-21. http://dx.doi.org/10.1177/0037768614560875

Michalowski, I. 2010. «Explaining the Accommodation of Religious Diversity in the Military: The US and Germany Compared». Pp. 397422 en 12th Berlin Roundtables on Transnationality. Cultural Pluralism Revisited: Religious and Linguistic Freedoms. Berlin: Irmgard Conninx Stiftung.

Molano, E. 1987. «La asistencia religiosa en los hospitales públicos». Pp. 321-38 en Dimensiones jurídicas del factor religioso: estudios en homenaje al profesor López Alarcón, editado por VV. AA. Murcia: Universidad de Murcia.

Moreno Antón, M. 2006. «La asistencia religiosa en España». Pp. 99119 en La libertad religiosa en España y Argentina, compilado por I. Martín Sánchez y J. G. Navarro Floria. Madrid: Fundación Universitaria Española.

Olmos Ortega, M. E. 1994. «La asistencia religiosa». Pp. 189-208 en Acuerdos del estado español con los judíos, musulmanes y protestantes. Salamanca: Universidad Pontificia de Salamanca.

Pérez-Agote, A. 2012. Cambio religioso en España: Ios avatares de la secularización. Madrid: Centro de Investigaciones Sociológicas.

Powell, W. W. y Jeannette A. Colyvas. 2008. «Microfoundations of institutional theory». Pp. 276-298 en The Sage handbook of organizational institutionalism, vol. 276, compilado por R. Greenwood, C. Oliver, K. Sahlin-Andersson y R. Suddaby. London: Sage Publications.

Prat, J. 2012. Els nous imaginaris culturals: Espiritualitats orientals, teràpies naturals i sabers esotèrics. Tarragona: Universitat Rovira i Virgili. 
Restrepo, D. A. y César Moreno. 2011. «La conversión religiosa en los centros penitenciarios: el caso de "La Blanca" de Manizales". Virajes 13: 237-255

Rostaing, C., Claire de Galembert y Céline Béraud. 2014. "Des Dieux, des hommes et des objets en prison. Apports heuristiques d'une analyse de la religion par les objets». Champ pénal/ Penal field 11.

Sointu, E. y Linda Woodhead. 2008. «Spirituality, gender, and expressive selfhood». Journal for the scientific study of religion 47: 259-276 http://dx.doi.org/10.1111/j.1468-5906.2008.00406.x

MAR GRIERA. Doctora en Sociología por la Universitat Autònoma de Barcelona (2009). Actualmente es profesora del Departamento de Sociología de la UAB y directora del grupo de investigación ISOR (Investigacions en Sociologia de la Religió) de la UAB. También es investigadora asociada al Centre de Sociologie des Religions et d'Éthique Sociale de la Universitat Marc Bloch (Strasbourg).
JULIA MARTíNEZ-ARIÑO. Doctora en Sociología por la Universitat Autònoma de Barcelona (2012). Investigadora postdoctoral en el Max Planck Institute for the Study of Religious and Ethnic Diversity (Alemania) y miembro de grupo de investigación ISOR del Departamento de Sociología de la UAB. Sus intereses son el judaísmo y la acomodación de la diversidad religiosa.

ANNA CLOT GARRELL. Licenciada en Sociología por la Universitat Autònoma de Barcelona (2010) y máster por la Lancaster University (2011). Actualmente, es doctoranda en Sociología en la Universitat Autònoma de Barcelona y miembro del grupo de Investigaciones en Sociología de la Religión (ISOR).

GLORIA GARCÍA-ROMERAL. Doctora en Sociología por la Universitat Autònoma de Barcelona. Colaboradora docente en el Máster de Análisis Político de la UOC e investigadora asociada al grupo de investigación ISOR de la UAB. Especializada en sociología de la religión, gestión política de la diversidad religiosa y comunidades islámicas. 